\title{
Recent Developments in Reliable and Reproducible Digital Immunogold Analysis in Electron Microscopy
}

\author{
Jens Krieger ${ }^{1}$, Martin Bartels ${ }^{1}$, Wei-Jun Chen ${ }^{1}$ and Michael Bode ${ }^{2}$
}

1: Soft Imaging System GmbH, 48149 Muenster, Germany

2: Soft Imaging System Corp., Lakewood, CO 80228, USA

Immunocytochemical techniques have evolved rapidly in biological and medical research and are very important static methods for understanding interactions in the cell. Establishment of immunological methods using multiple antibodies for multiple epitopes together with multilabelling - using gold probes of different size in the same sample - is now possible for virtually every biological laboratory by using high quality protein-gold complexes and certain antibodies which are available commercially in a broad range and in high-quality. Together with this possibility comes the need to analyze the results in the same high quality - reliably and reproducibly.

Immunogold probes are available in sizes ranging from about 1-40 $\mathrm{nm}$ for electron microscopy. While the resolving power of a transmission electron microscope is much better than the smallest size, the question arises how to record the results and how to analyze them.

We are presenting a hardware - software combination using current digital techniques for almost all types of TEM. The resolution power of the 11 Mega Pixel CCD camera Morada in combination with the software Solution EMarker for iTEM provide both reliability and flexibility for fast automated analyzing and archiving of digital images of immunogold labeled samples. Overview images of around 10k magnification can easily be analyzed, as well as detailed views of tissues. It is future safe in its possibility not only to be used on standardized labeled samples with single labels: both double-labeling and any multi-labeling probes can be assayed with the same simplicity and reliability and moreover it is not restricted to immunogold assays only. 